\title{
ÉDITORIAL
}

\section{Pronostic et prédiction de la réponse aux traitements par la biologie des cancers : une opportunité pour changer les concepts par l'innovation}

\author{
Y. Belkacémi ${ }^{1,2, a}$, J. Gligorov ${ }^{3, a}$ \\ ${ }^{1}$ Service d'oncologie-radiothérapie, hôpital Henri-Mondor, 51, avenue du Maréchal-de-Lattre-de-Tassigny, \\ F-94000 Créteil cedex, France \\ ${ }^{2}$ Faculté de médecine de Créteil, université de Paris-XII, 8, rue du Général-Sarrail, F-94010 Créteil cedex, France \\ ${ }^{3}$ Service d'oncologie médicale, AP-HP, hôpital Tenon-Cancer-Est, université de Paris-VI, Paris, France \\ ${ }^{a}$ Association de radiothérapie et d'oncologie de la Méditerranée (Arome ; www.aromecancer.org) \\ Correspondance : yazid.belkacemi@hmn.aphp.fr
}

Biologie des cancers, ce numéro spécial porte un titre (au pluriel) qui signifie bien le défi du chercheur face à la diversité et à la complexité du processus tumoral depuis sa genèse jusqu'au constat de l'échappement aux moyens thérapeutiques dont nous disposons. Cependant, nous avons la certitude que la recherche biologique va continuer d'apporter aux cliniciens un arsenal de nouveaux paramètres capables de (re)définir le pronostic d'une maladie et/ou d'envisager l'individualisation d'une stratégie thérapeutique dès le diagnostic. Dans ce domaine la citation : "les vérités d'un jour ne sont pas celles de toujours!", est plus que jamais d'actualité.

$\mathrm{Au} x \mathrm{xl}^{\mathrm{e}}$ siècle, les perspectives de la recherche en cancérologie sont rythmées par les avancées enregistrées et mises à disposition en un temps record par les réseaux d'informations de plus en plus développés. Cependant, pour le clinicien, ces avancées restent au stade de "l'invention " et ne peuvent en aucun cas être considérées comme des " innovations " tant qu'elles ne bénéficient pas aux patients et à la pratique clinique. En effet, l'innovation biomédicale ne peut être considérée en tant que telle que lorsqu'elle correspond à un résultat concret (une nouvelle méthode diagnostique ou un nouveau produit) utilisé par les praticiens au bénéfice de leurs patients. Dans ce contexte, "le savoir que l'on ne complète pas chaque jour diminue tous les jours " et la nécessité d'une mise à jour des connaissances est un prérequis pour orienter et avancer en recherche appliquée afin d'offrir aux patients les meilleurs traitements possibles adaptés à leurs états physiologiques (l'hôte) et à leurs tumeurs.

L'autre prérequis, beaucoup plus général, est d'avoir la conviction que l'innovation ne peut résulter que d'une recherche fondamentale bien menée pendant de longues années où les mots clés restent la persévérance, l'interdisciplinarité et l'espace de liberté pour le chercheur. Au vu de ces prérequis, on ne peut que conclure à l'importance de l'initiation à la recherche des jeunes très tôt dans leurs carrières avec en prime des perspectives valorisant leurs efforts et une reconnaissance de leurs contributions aux chalenges de la recherche translationnelle. Victor Hugo disait : " la beauté pour les choses et la reconnaissance pour les hommes".

Ce numéro spécial est justement dédié à des articles de biologie écrits par de jeunes oncologues médicaux et des oncologues-radiothérapeutes en formation. II constitue un recueil de mises au point abordant les thématiques des sujets de thèse ou d'autres travaux menés par ces jeunes au cours de leur initiation à la recherche.

Les deux mots clés de ce numéro sont le pronostic et la prédiction de réponse aux traitements. Les auteurs ont abordé la perspective d'une individualisation des traitements selon le pronostic défini par des techniques et des méthodologies nouvelles évaluant la sensibilite aux traitements des tumeurs, des cellules souches cancéreuses et des tissus sains pour un meilleur ratio bénéfice/risque. Concernant la prédiction de réponse, certains de ces articles ont abordé quelques marqueurs susceptibles de devenir incontournables avant la prescription d'une thérapie ciblée. Ainsi, ce type de démarche nous paraît fondamental pour encourager les plus jeunes à entrer dans cette nouvelle ère d'une recherche biologique appliquée où les avancées récentes montrent qu'il n'y a jamais eu autant d'opportunités pour innover. Cela est vrai à la condition d'être persuadé que "l'innovation passe par la volonté de considérer le changement comme une opportunité ", ...et ce, à tous les niveaux de décision. 